\title{
Le prix des esclaves dans les comédies latines
}

\section{Marie Delcourt}

\section{Citer ce document / Cite this document :}

Delcourt Marie. Le prix des esclaves dans les comédies latines. In: L'antiquité classique, Tome 17, fasc. 1, 1948. Miscellanea Philologica Historica et archaelogia in honorem Hvberti Van De Weerd. pp. 123-132;

http://www.persee.fr/doc/antiq_0770-2817_1948_num_17_1_2833

Document généré le 24/01/2017 


\title{
LE PRIX DES ESCLAVES DANS LES COMÉDIES LATINES
}

\author{
par Marie Delcourt
}

Dans son Histoire de l'esclavage dans l'antiquité (1), Henri Wallon a réuni les documents que l'on connaissait de son temps relativement au prix des esclaves. A l'époque grecque, dit-il, les prix moyens étaient de

2 mines vers le temps de la guerre médique,

3 mines entre celle du Péloponnèse et l'époque alexandrine,

4 à 5 mines sous les diadoques.

La seconde de ces périodes offre déjà assez de textes pour que l'on puisse distinguer, parmi les esclaves, des spécialités. On donne volontiers 2 mines ou 2 mines $1 / 2$ pour un mineur ou un manœuvre, 3 à 4 pour un artisan, 5 à 6 pour un bon contre-maître ou un domestique d'intérieur, 10 à 15 pour des hommes particulièrement instruits et intelligents, 20 à 30 mines, dit Wallon, "pour les esclaves mis au service du luxe et du plaisir ", et il ajoute que, dans cette catégorie, il n'y a aucune limite.

Ces prix ont été confirmés pour l'époque hellénistique par les papyrus que W.-L. Westermann a consultés pour écrire, en 1935, l'article Sklaverei dans le VIe supplément de la Realenzyklopedie. Une petite fille de sept ans est vendue à Birta 50 drachmes, un jeune garçon, vers $258,112 \mathrm{dr}$., une esclave et sa fille, vers 259 , $200 \mathrm{dr}$. par, tête, une jeune fille $300 \mathrm{dr}$. La prime pour la capture des esclaves marrons était à la même époque, en Palestine, de $100 \mathrm{dr}$., Hérondas donne 3 mines pour le prix d'un esclave à Cos (2).

Les tarifs d'affranchissement et de rançon sont plus élevés, ce qui se comprend. Dans les deux cas, le détenteur de la marchandise humaine sait qu'il peut forcer le prix sans décourager l'ac-

(1) Tome I, p. 119 ; tome II, p. 159, 1879.

(2) VI, 21, Crusius. 
quéreur. En Grèce centrale, entre 201 et 50 avant J.-C., un esclave se rachète pour une somme qui est en moyenne de 3 à 5 mines et qui va parfois jusqu'à 10 et même 20 mines. La convention entre Démétrios Poliorcète et les Rhodiens en 304 fixe la rançon à 5 mines par prisonnier ( $\left.{ }^{1}\right)$. C'est le prix auquel Annibal vendit en Achaïe 1200 prisonniers romains (2). Le même Annibal, après avoir exigé une rançon fort élevée pour les prisonniers de Cannes, finit, semble-t-il, par se contenter d'une livre et demie d'argent, c'est-à-dire environ 150 drachmes : le Carthaginois devait ne savoir que faire de ses prisonniers et il aura "bradé " la plus mauvaise partie du stock, celle qu'on tardait le plus à racheter. Cela explique la protestation des soldats romains contre l'achat et l'enrôlement d'esclaves dans l'armée : ils font valoir qu'il n'en coûterait pas davantage de racheter une partie des prisonniers faits par Hannibal $\left({ }^{3}\right)$.

Deux renseignements relatifs à Caton indiquent que le prix des esclaves d'un bon rendement était élevé dans la liome du ${ }_{11}{ }^{\mathbf{e}}$ siècle. Lorsqu'il fut censeur et chargé d'établir l'impôt, Caton frappa d'un coefficient 10 , comme d'une taxe de luxe, les esclaves qui avaient coûté plus de 10.000 as, soit un peu moins de neuf mines. Lui-même ne voulait pas d'esclaves de luxe, mais seulement des hommes intelligents et travailleurs; il n'en paya jamais aucun plus de quinze mines.

Avec les comédies latines, nous entrons dans un monde différent, où apparaissent tout de suite des prix beaucoup plus élevés. Exagération comique, dit Wallon. Clichage, dit Westermann, qui ajoute : "Les prix mentionnés dans les comédies proviennent d'une exagération propre à ce genre, remontant à l'original grec; ils sont sans valeur pour l'établissement des prix réels, aussi bien en Grèce qu'à Rome * ( $\left.{ }^{4}\right)$.

(1) DIOD., III, 84, 6.

(2) Tite-live, xxxiv, 50, d'après Polybe et Plut., Flam., 13.

(3) Tite-Live, xxir, 58, 59 ; Plut., Fabius, 7 ; Aulv-Gell.e, vil, 18.

(4) * Diese Preise beruhen auf der typologischen komischen Uebertrcibung in den griechischen Originalen und sind als Beleg fur wirkliche Preise sowohl in den griechischen Stadten wie auch in Rom wertlos". 
Ces jugements sans nuances demandent à être vérifiés.

Les esclaves mis en vente dans la comédie sont presque toujours des femmes; l'acquéreur est presque toujours un amant, décidé à payer le prix que le marchand demandera ; celui-ci est un leno, qui loue ses pensionnaires en attendant de les vendre, et qui en retire ainsi sans délai un appréciable loyer. Par conséquent, les prix seront toujours surfaits, ce qui ne veut pas dire qu'ils soient inauthentiques. Nos petits-fils regarderont avec étonnement celui qui leur dira qu'en 1944 il a vendu un tableau d€ maître pour acheter 2 kilos de café. Un économiste qui, dans deux millénaires, recueillera ce renseignement se gardera d'en tirer une indication moyenne, mais il fera prudemment de ne pas le rejeter a priori comme wertlos. La comédie nous donne presque toujours des cas exceptionnels, mais l'exception est toujours psychologiquement fondée et il n'y a aucun prix excessif qui n'apporte une indication sur un caractère.

Dans un certain nombre de cas, des renseignements donnés dans le cours du récit, sans aucun affectus sensible, sont vraisemblables. Dans les Prisonniers de guerre (974-979), l'esclave favori Stalagmus s'enfuit en enlevant le fils de son maître, qu'il vend six mines à Elis. Dans le Petit Carthaginois (896), une nourrice est prise avec deux petites filles puniques de 4 et 5 ans par un pirate sicilien, qui vend tout le lot pour 18 mines à un leno de Calydon, en prévenant l'acheteur que les deux enfants sont libres. Étant donné le risque de se voir enlever la marchandise sans compensation, le leno payait là un prix assez élevé, mais il devait être rassuré par le dépaysement. L'officier du Rustre (529), estimant a parte (c'est-à-dire sans vantardise) la valeur de ses cadeaux, dit que deux servantes, une robe de Phrygie, une autre de pourpre tyrienne et des fourrures du Pont valent ensemble 20 mines. C'est dans un aparté aussi que le leno du Charançon (528) dit avoir payé une fillette 10 mines. Phédria (Eunuque, 169) offre à sa maîtresse Thaïs une jeune fille et un eunuque qu'il a payés ensemble 20 mines; il mentionne le prix avec une certaine emphase, comme un homme qui n'est point regardant lorsqu'il s'agit de faire un beau cadeau. Mille drachmes sont donc un prix élevé pour un ou une esclave adulte. Ce prix est cependant dépassé dans le Persan, où Toxile, esclave lui-même, cherche à se procurer 600 nummi pour racheter sa bonne amie. Si, comme tout le donne à penser, 
le nummus de Plaute équivaut au didrachme $\left({ }^{1}\right)$, la somme est forte : le maître sait qu'il peut tenir la dragée haute à l'esclave amoureux; d'autre part, la pièce ne tient que si Toxile a besoin d'une forte somme, assez inaccessible pour le faire recourir aux grands moyens. Lorsque le leno du Cordage (1406) déclare qu'il a acheté Ampelisca 1.000 nummi, c'est-à-dire 20 mines, il ment; Démonès le sait et le public aussi. Ces renseignements sont confirmés par l'histoire compliquée et assez confuse de la courtisane volée qui remplit les premières scènes des Frères de Térence. Le leno prétend l'avoir achetée 20 mines et entend la revendre avec bénéfice ; mais les ravisseurs l'étourdissent pour obtenir un rabais. Eschine suggère que la jeune fille est libre, mais Sannion ne se trouble pas; le Syrien a su, Dieu sait comment, que le leno est pressé de partir pour Chypre, où il a de gros intérêts ; il lui offre alors 10 mines, et Sannion avoue (244) : etiam de sorte nunc venio in dubium miser, c'est-à-dire : Ah ! ma guigne! voici même le principal mis en question. Il est inutile de se demander si sors désigne le dernier prix d'achat ou le prix de revient: Sannion estime que 10 mines ne lui laissent aucune marge de bénéfice. Il n'y a rien à tirer du passage des Prisonniers de guerre (353) où Hégion demande une garantie de 20 mines en cas d'évasion de Philocrate : ce n'est pas un prix de vente, c'est une caution.

En dehors de ces cas, les prix sont toujours exagérés. Ce n'est pas Plaute qui les grossit à plaisir, mais bien les passions de ses héros. La seule mention d'un prix offert ou demandé était, pour les auditeurs, une indication psychologique qui provoquait le rire. Lorsqu'un personnage accepte de donner 60 mines pour une esclave, le public s'amusait de lui, comme il s'amuserait si, dans une farce mettant en scène le temps du rationnement, quelqu'un offrait à un fraudeur 2.000 francs pour un kilo de beurre : "Fallait-il qu'il aimât le beurre l" Et si par surcroît le personnage est antipathique, si le public sait ou devine que la marchandise lui sera enlevée sans compensation, la joie n'a plus de bornes. C'est le cas dans

(1) W. Schwabacher, s. v. Nummus dans le XXXIV vol. de la Realenzyklopedie, renvoie aux articles de Mattingly et Robinson d'une part, de Tenney Frank d'autre part. Dans les deux seuls cas dont j'aie à m'occuper ici, le sens du mot ne paraft pas douteux. La pire des méthodes est probablement de masquer la difficulté en traduisant tantôt par denier, tantôt par écu (cf. Cordage éd. Ernout, 1323 et 1406), c'est-à-dire par des mots vieillis dont le sens est devenu confus pour nous. 
le Persan, où le leno donne une somme énorme pour une jeune fille qui est libre et qu'il devra restituer.

Dans les comédies qui ont pour centre un jeune homme amoureux d'une esclave, le leno demande en général de 20 à 40 mines. Ballion (le Fourbe, 51 sqq.) obtient d'un officier 20 mines pour Phénicie, et Callidore, pour la souffler au premier acquéreur, est bien obligé de trouver ou d'escroquer la même somme. Ce jeune fou de Philolachès (le Revenant, 300 ) a dû donner 30 mines pour affranchir Philématie. Phédria (Phormion, 556) a besoin d'en avoir autant pour acheter son amie; ses camarades trouvent la belle un peu chère, mais lui estime qu'à ce prix elle est pour rien. Le leno du Charançon (63) demande tantot 30 mines, tantót un talent pour Planésie, car les prix montent dès qu'il y a plusieurs amateurs, et ici, comme dans le Fourbe, c'est un officier qui provoque la surenchère. Au surplus, comme l'amant n'a en mains ni 60 mines, ni 30, l'écart entre les prix l'inquiète assez peu. En fait, Planésie est vendue 30 mines, plus 10 pour la toilette et les bijoux (343). Quoique ces prix soient excessifs, le leno ne fait pas une si bonne affaire, puisqu'il l'a payée 10 mines quand elle était petite, que dans l'intervalle elle est restée vierge, et qu'il n'a donc tiré d'elle aucun profit. Stratippoclès, dans Epidicus (52 et 296), paie 40 mines à une vente, pour une jeune fille dont il est fou, ce qui lui vaut de tomber dans les griffes d'un usurier : fredaine d'officier fils de famille. Son père est disposé à mettre le même prix, et même 50 mines, pour une musicieñne qu'il croit être sa fille. La scène des enchères, dans le Marchand (421-434), prouve que 20 mines, pour une belle fille, sont une somme qu'on demande seulement à un homme épris. Mais une mère avisée loue pour 20 mines la jouissance de sa fille pendant un an, et cédera la nue propriété pour 2 talents (le Prix des ânes, 89, 231 et 193). On voit avec quelles corrections il faut accepter la proposition de Louis Havet, lorsqu'il dit dans sa préface à la pièce : « Ni Térence, ni Maccus ( ${ }^{1}$ ne pouvaient proposer à leur public que des prix valables pour leur temps et valables pour Rome. Et les prix romains et grecs, d'ailleurs, ne pouvaient différer sérieusement, car l'exportation des femmes était chose courante. En somme, entre le temps de Térence et celui de Maccus, le commerce des courti-

(1) Louis Havet attribue l'Asinaria, non à Plaute, mais à un hypothétique Maccus. 
sanes avait évolué." Cette opinion, diamétralement opposée à celle de Westermann, est également trop simple. Le prix d'une courtisane dépend de l'habileté du marchand, du désir et des ressources de l'acheteur. Dans le monde moderne, le profit va à l'intéressée, dans le monde ancien il allait d'abord au leno, quoique la Cléérète du Prix des ânes soit une Madame Cardinal qui sait son métier. Les prix excessifs sont destinés à diriger les sentiments des spectateurs, à ridiculiser l'amant jobard, à rendre odieux le leno féroce et avide, à rendre admissible la spoliation dont il sera finalement l'objet. Prétendra-t-on qu'un cuisinier, vers l'an 200, valait 30 mines, parce que, dans la Marmite (309-310), Anthrax demande, en parlant du pauvre et avare Euclion :

Censen talentum magnum exorari pote

$\mathrm{Ab}$ istoc sene, ut det qui fiamus liberi?

La question d'Anthrax n'est pas une vantardise, comme le croit Wallon, mais une énorme naïveté qui faisait rire tout le petit peuple. Celui-ci s'amusait aux dépens de ces fils de famille toujours désargentés, toujours obligés de payer trop cher une marchandise dont le poète lui disait le peu de valeur véritable. Ces indications relatives au prix des courtisanes ont exactement la même importance pour un économiste que le chiffre des revenus d'une I' milienne d'Alençon ou d'une Liane de Pougy; personne ne songera à en rien inférer relativement aux salaires des rats de l'Opéra, ni même des danseuses étoiles, mais aucun historien ne les négligera dans un tableau des années 1900. De plus et surtout, les prix pratiqués dans les comédies éclairent de curieux détails de psychologie et de poétique. J'en prendrai deux exemples, dans le Persan et dans le Cordage.

Le leno du Persan paie la fausse Orientale 60 mines (on lui demande encore, pour couronner le tout, 10 mines pour le trousseau, qu'il refuse cependant), somme absurde, insensée, car il n'a sous la main aucun amateur à qui revendre la marchandise avec bénéfice. C'est, dans toutes nos comédies, le seul prix absolument invraisemblable. Un homme amoureux peut payer n'importe quoi à un leno pour affranchir la fille qu'il aime; mais un leno ne donne pas n'importe quoi pour acquérir une esclave qui lui restera longtemps sur les bras. Cette exagération prouve que la pièce, qui est une opérette au moins autant qu'une comédie, devait être jouée en farce. L'énormité de la somme, la jobardise du leno divertissaient le public, et l'étrangeté du costume faisait passer la muscade. 
Plus important est le problème du Cordage, dont le dénouement était très clair pour les spectateurs, mais a été mal compris par la plupart des modernes.

Le vieil esclave Gripus a retrouvé la valise perdue par le leno I abrax ; elle contient la valeur d'un talent; après quelques péripéties, elle arrive entre les mains de Démonès maître de Gripus, non sans être revendiquée par le leno. Démonès arbitre la situation : il donne 30 mines à Labrax, à charge d'affranchir Ampelisca que le leno prétend avoir coûté 1000 nummi, soit 20 mines (en quoi, évidemment il ment) ; il garde 30 mines pour lui et, en échange, il affranchit le pauvre Gripus.

Qu'est-ce à dire? Labrax et Démonès font l'un et l'autre une excellente affaire, car ni Ampelisca, qui n'est aimée que d'un autre esclave, ni Gripus qui est vieux ne valent 30 mines. Gripus est volé et ne cache pas sa déception. Sur quoi Démonès invite le leno à souper.

Démonès, présenté au début de la pièce comme un personnage sympathique est donc un parfait hypocrite. Deux indications de prix suffisaient à renseigner les spectateurs sur le faux bonhomme. Je ne vois pas un seul annotateur moderne qui en ait tiré parti.

Celui qui a un peu pratiqué Plaute ne s'étonnera pas de cette terrible impartialité. Plaute ne permet pas au spectateur de se solidariser avec un ou plusieurs de ses personnages. C'est Molière qui met au centre du théâtre un couple d'amoureux dont l'appétit de bonheur révèle les ridicules ambiants, car le test essentiel du ridicule c'est qu'il empêche le bonheur. Plaute n'a pas de personnages sympathiques: ses amoureux sont niais et incapables, ses jeunes filles sont stupides et coquettes, ses pères nobles sont des grigous qui n'hésitent jamais devant une indélicatesse. Dans les Frères, la pièce où Térence suit du plus près l'esthétique plautienne, il en va de même : Micion est aussi ridicule que Déméa, mais il ne l'est pas au même moment.

Ce qui est dit ci-dessus des prix payés par des fils de famille pour affranchir leurs amies jettera peut-être quelque lumière sur les sommes très élevées que Martial et Pline affirment avoir été payées pour des jeunes garçons. Westermann se refuse à les admettre et allègue des corruptions du texte. Scepticisme bien im- 
prudent. Lorsque nous lisons qu'on a donné 100.000 sesterces pour un beau garçon, gardons-nous bien de faire entrer ce chiffre dans le calcul d'une moyenne, mais ne le rejetons pas. Soyons sûrs qu'il s'agit d'un de ces "mignons" qui font déjà leur apparition chez Plaute - mais non chez Térence - et dont les critiques ont trop peu signalé la présence.

Fn effet, le mot latin puer ne correspond nullement au $\pi a i \varsigma$ des comédies grecques, qui peut se dire d'un esclave de n'importe quel âge (comme notre garçon appliqué à un serveur de restaurant), si bien que Ménandre, à quelques vers d'intervalle, l'applique à un bébé et à un serviteur adulte. Puer chez Plaute signifie mignon. Dans le texte, un esclave adulte est désigné par son nom, ou, lorsque son interlocuteur ignore qui il est, par le mot adulescens. Le vocatif puere est parfois adressé, sans affectus, à des enfants d'escorte qui jouent le rôle muet de pages (Prix des ânes, 382, 891 et quelques autres cas analogues). Dans les listes de personnages, les esclaves sont toujours dits servi (1) sauf quand ce sont des mignons au nom parlant comme le Pinacium - Tableautin - de Stichus, le Paegnium - Joujou - du Persan, le prostitué anonyme du Fourbe (768) qui, dans un monologue déchirant, se plaint de sa malechance. Tous sont annoncés comme pueri et le sens du mot est parfaitement clair, souvent précisé par quelque geste, une allusion, un compliment appuyé (Persan 192, 202, 204, 209). Joujou est le puer Toxili, non pas l'esclave de Toxile (qui est esclave lui-même) mais son chéri, comme Tableautin est celui de Gélasime (Slichus, 270). Le Ballion du Fourbe prostitue des garçons aussi bien que des jeunes filles et lui-même a un mignon auquel il parle avec tendresse, dans la terrible scène des menaces (132 sqq., notamment 170, 241), en l'appelant puere. Une " mimique ionienne " achevait évidemment d'éclairer les spectateurs.

Il faut se souvenir de ces passages pour comprendre le dénouement des Prisonniers de guerre. Stalagmus a été le mignon de Hégion et, même repris, même convaincu de vol et promis à un châtiment sévère, il lui parle sur un ton insolent qui n'est pas le fait d'un esclave ordinaire (954 squ.). Ici encore, comme dans

(1) Mème libanus - Brindencens -.. dans le Prix des ines, malgré son nom parlant et la description qui est faite de lui au vers 627. Mais il ne joue aucun rôle de mignon dans la pièce. La désignation puer dans les listes n'est donc employée qu'avec intention. 
le Cordage, Plaute détruit la sympathie qu'il avait d'abord inspirée pour son personnage. Hégion comme Démonès est un hypocrite. S'il a perdu son fils, c'est pour avoir trop accordé à un esclave favori.

Je ne vois chez Térence qu'une seule allusion de ce genre, et très brève, à propos de Thrason dans l'Eunuque (479, clause de style lorsqu'il s'agit d'un officier, comme le prouve Soldat Fanfaron 1101). Térence n'emploie presque jamais le mot puer dans le sens de petit page (excepté Phormion 152, Belle-mère, 359, 409, 719); il dit plus volontiers, peut-être pour éviter toute équivoque, servulus (Bourreau de soi-même 191).

Un seul passage nous donne une indication concernant le prix des mignons. Le fils enlevé d'Hégion, dans les Prisonniers, a été vendu 6 mines, ce qui est beaucoup pour un petit enfant, sous le nom parlant de Paegnium. Mais l'acquéreur n'a pas voulu le livrer à la débauche, il l'a débaptisé, lui a donné un nom décent et a fait de lui le camarade de son fils Philocrate qui déclare (991-2) :

is mecum a puero puer

bene pudiceque educatust usque ad adulescentiam.

C'est exactement la formule par laquelle on annonce qu'une jeune fille vendue est restée vierge, ce qui prélude invariablement, comme ici aussi du reste, à une reconnaissance. La parallélisme est assez frappant.

Cette enquête nous apprend plusieurs choses.

Nulle part il n'y a de "clichage comique". Les prix sont extrêmement variables et chacun dépend de circonstances que le poète ne manque jamais d'indiquer.

Les prix mentionnés sans affectus, en monologue et d'une façon désintéressée, sont parfaitement vraisemblables.

La plupart des autres sont surfaits pour des raisons qui tiennent aux passions des acquéreurs. Ils renseignent les spectateurs sur la psychologie des personnages. Wallon avait raison de dire qu'en matière de luxe et de plaisir il n'y a pas de limites aux prix.

Une seule exagération invraisemblable: le prix de la fausse Orientale, dans le Persan, crée une atmosphère de bouffonnerie. 
Enfin, les bourgeois (1) de Plaute sont aussi cupides que les lenones, aussi débauchés que les jeunes gens.

(1) N'est-ce pas ainsi qu'il faudrait rendre l'intraduisible senex? car la femme du senex est simplement appelée mulier, jamais anus. -- Je ne veux pas terminer cet article sans remercier mon ami Gaston I,ibon, de la IBibliothdque royale, de l'aide précieuse qu'il a bien voulu m'apporter. 